\title{
FAMILIE, VERWANDTSCHAFT UND FREUNDSCHAFT BEI DEN OLO NGADJU IN S. O. BORNEO.
}

VON

F. GRABOWSKY.

Es war mir sehr interessant bei den Olo Ngadju ausser den uns gelaüfigen Bezeichnungen für Verwandtschaftsgrade auch solche anzutreffen, die unserer Sprache entweder ganz fehlen oder durch weitläufige Umschreibungen ausgedrückt werden müssen. Es schien mir daher der Mühe werth, die sämmtlichen Bezeichnungen, die Familien-, Verwandtschafts- und Freundschaftsverhältnisse der Olo Ngadju (Dajacken) betreffen, zu sammeln und das Resultat dieser rein compilatorischen Arbeit zu publicieren.

Die Dajacken unterscheiden wie wir "nahe Verwandte, kula" und "entfernte Verwandte oder kakula."

Eine Heirath bildet auch bei ihnen die Vorbedingung zur Gründung einer Familie. Allerdings folgen die jungen Leute selten eigener Neigung, sondern die beiderseitigen Eltern verabreden das Nöthige; sie wollen "sanger" werden d. h. jhre Kinder mit einander verheirathen. Wir besitzen keinen Namen für dieses auch "sanger djambulau " oder "nampah" genannte Verwandtschaftsverhältniss zweier Schwiegerväter oder Schwiegermütter unter einander.

Sind die Praeliminarien zu einer Heirath glücklich erledigt, so wird der Jüngling "Bräutigam" "bakal banae" d.h. wörtlich: Material für einen Ehemann. Er soll heirathen "masawae". Während wir nun nur einen Ausdruck für beide Geschlechter dafür besitzen, sagt der Dajacke von einer Jungfrau, die heirathet, sie "babanae".

Sobald die Brautleute "Hochzeit gehalten" "sawae habanae" 1 sind sie "sawä" Ehefrau und "banä" Ehemann geworden, und treten

$1 \mathrm{Ob}$ der Umstand, dass nach dajackischer Sitte der Mann der Frau folgt, schon durch-die Stellung von habanae hinter sawae, in dem Ausdruck für "Hochzeit halten" "sawae habanae", ausgedrückt werden soll, bin ich nicht im Stande anzugeben, halte es aber wohl für möglich.

5 Volgr IV. 
dadurch in ein neues verwandtschaftliches Verhältniss "pandjalah" zu den beiderseitigen Familien.

Sie nennen ihre gegenseitigen Eltern nun "empo", was sowohl Schwiegervater als Schwiegermutter bedeutet. Will man die "empo" noch besonders als Respectspersonen hervorheben, so nennt man sie "bakasan" oder "empo bangkasan". Die Geschwister der Schwiegereltern nennt man "empo lambong", wofür wir auch kein Wort besitzen. Die Schwiegereltern nennen den Schwiegersohn "manantu hatuae", die Schwiegertochter "manantu bawi". - Schwester und Frau des Mannes werden "Schwägerin" "iwan". Nur der Mann, nicht seine Geschwister, wird "ajup", Schwager der Geschwister seiner Frau und umgekehrt. Das verwandtschaftliche Verhältniss der Geschwister des Mannes zu denen der Frau wird durch "pahari" d. h. Brüder oder Schwestern, bezeichnet.

"Hat ein Mann zwei oder mehrere Frauen so nennen dieselben sich untereinander "djambua".

Zwei Männer welche zwei Schwestern, oder Frauen welche zwei Brüder geheirathet haben, reden sich mit "duäi" an. Man nennt ein solches Heirathen. "hasansulang sawae d. h. an einander vorbei heirathen."

Wird die Ehe mit Kindern gesegnet, so ergeben sich folgende Verhältnisse. Die Schwiegereltern bekommen "aeso", Enkel, Kleinkinder, von denen sie "ambi" 1 Grossmutter und "buae" 2 Grossvater genannt werden.

Die Kinder "anak" oder "anak olo" sind entweder Söhne "anak hatuä" oder Töchter "anak bawi " - Awau oder anak awau ist eine zärtliche Benennung für Säuglinge beiderlei Geschlechts.

Bevor die Knaben einen bestimmten Namen erhalten haben, ruft man sie "atak".

Auch "lungus oder dagau (Hahn) sind zärtliche Benennungen für "Knabe, Sohn" .

"Apang" nennen grössere Kinder den eigenen Vater: "aba", Vater wird von kleineren Kindern und zu kleineren Kindern von ihrem Vater sprechend, gebraucht; den Vater anderer nennt man "bapa". "Indu" nennen die Kinder die Mutter." 3

"Der Aelteste" unter mehreren Geschwistern ist der "tambakas".

1 Die Grossmutter anderer nennt man "tambi ".

2 Buae nennt man auch den Vater der Stiefeltern, den Vater der eigenen oder Stiefeltern der Frau und alle Brüder dieser Grossväter.

3 Bei der Namengebung des ersten Kindes, ändern die Eltern ihre eigenen Namen 
Der Bruder nennt seinen Bruder ebenso wie die Schwester ihre Schwester im Algemeinen "pahari". Der Bruder nennt die Schwester "betau", die Schwester nennt denn Bruder "njaha". Der jüngere Bruder oder die jüngere Schwester heist "andi". Von ihren jüngeren Geschwistern werden der ältere Bruder oder die ältere Schwester "aka", von andern Menschen "kaka" genannt.

Für die Brüder oder Schwestern des Vaters oder der Mutter wird man, wie bei uns, Neffe oder Nichte "aken" nnd nennt den Oheim "ama", den Oheim eines Andern "mama", die Tante "minna". Auch die Basen der Eltern nennt man Tante und giebt diesen Namen auch alten Frauen als Ehrennamen, ebenso wie man andere, ältere Männer, welche nicht wirklich Verwandte sind, aus Achtung oft "ama" nennt.

"Stirbt Vater oder Mutter, so wird das Kind "nulae" Waise; entweder "nulaen indu", wenn die Mutter stirbt oder "nulaen hapa" wenn der Vater stirbt; "nulae pahatu" sind Vollwaisen. Heirathet ein Mann eine Wittwe mit Kindern so wird er deren Stiefvater "bapa tiri”; eine Stiefmutter heisst "indu tiri”; die Stiefkinder sind "anak tiri".

Hat eine Frau von ihrem zweiten Mann auch Kinder, so heissen diese "pahari oder betau sapak piak" d. h. Brüder oder Schwestern, welche dieselbe Mutter aber verschiedene Väter haben, im Gegensatz zu "pahari oder betau idjä kalambutau" d. h. rechter Bruder oder rechte Schwester; oder Stief bruder und Stiefschwester, welche denselben Vater (aber andere Mütter) haben.

Das Verwandtschaftsveahältniss der Neffen und Nichten zueinander wird ebenfalls auf die Grosseltern zurückgeführt, so sind z. B. "aken idjä tato" Neffen im zweiten Gliede" d. h. deren Väter rechte Neffen waren. Die Dajacken zählen bis "aken telo tato", Neffen in vierten Gliede. Bei verwandtschaftlichem Verhältniss durch Geburt findet aber die reciproke Bedeutung wie bei "ajup" Schwager nicht statt. "Pahari oder betau idjä tato" d. h. Brüder oder Schwestern eines Grossvaters sind demnach rechte Vettern oder Basen, welche denselben Grossvater haben; "pahari oder betau duae tato" Kleinvettern, die denselben Urgrossvater haben.

um. Hiess z. B. der Mann "Djalan", die Frau "Budha" und sie nannten ihr erstgeborenes Kind etwa "Linda" (Mädchenname), so nennt sich der Vater fortan : "Palinda" d. h. Vater der Linda und die Mutter "Indulinda" d. h. Mutter der Linda. - Ein Kind darf den Namen seines Vaters nicht aussprechen, das ist "pali», unerlaubt; er ersucht Andere für ihn zu antworten. 
"Tato" eigentlich "Ưrgrossvater" wird oft für "bnae" Grossvater gebraucht.

"Tato hiang" sind die Vorfahren und deren Nachkommenschaft wird mit "anak" djaria(n) oder "panakan" bezeichnet.

Bleibt eine Ehe kinderlos, so nehmen die Eheleute meistens ein elternloses Kind als ihr eigenes an, sie adoptiren es. Ein solches Kind heist "anak laku" oder "anak induan", wird wie das eigene Kind aufgezogen und tritt auch in dieselben Rechte ein.

Es ist aber bei den Dajacken auch eine sehr verbreitete Sitte Kinder nur proforma zu adoptiren; solche Kinder nennt man "anak ambai", "anak angkat" oder "ambai ango". Dieses Adoptiren geschieht nur um den Bekanntenkreis zu vergrössern; natürlich wohnen solche adoptirte Personen nicht zusammen. Die Adoption geschieht, nachdem man tüchtig gegessen und getrunken dadurch, dass von beiden Personen etwas Blut auf ein Sirihkausel gethan und also gekaut wird. Das Blut wird von den Männern, welche adoptiren und von allen zu Adoptirenden von der linken Schulter, von adoptirenden Frauè aus der linken Brust genommen. Solche Personen, von denen der eine das Kind des andern adoptirt hat, nennen sich "Dampo."

Nur selten kommt es vor, dass eine dajackische Jungfrau unverheirathet bleibt; man nennt sie in diesem Falle "budjang manohos" d. h. verfaulende Jungfrau", unserer "alten Jungfer" entsprechend.

Um so häufiger kommt er vor, dass Kinder vor der Eheschliessung eintreffen. Diese Kinder nennt man "ampang", anak ampang. oder "sarau."

Der Dajacke darf seinen Namen nicht nennen; will er nun Jemand, der denselben Namen mit ihm führt, anreden oder rufen so bedient er sich der Worte "antai oder pangantai, etwa unserm "Namensvetter" entsprechend.

Es bleibt uns nun noch übrig die freundschaftlichen Verhältnisse zu erwähnen. Dajackische Männer, die befreundet sind, nennen einander "ulae", dajackische Frauen "aring". Seinen malaiischen Freund nennt der Dajacke "urai oder urai unda, den chinesischen "tangkai".

Den Frauen reicher und vornehmer Leute giebt man den Ehrennamen "njai"; ihre Töchter werden mit "nai" angeredet, etwa unserm "gnädige Frau" und "gnädiges Fräulein" entsprechend.

"Njonja" endlich ist der Ehrenname für Frauen der Europäer, während diese mit "tuan" d. h. Herr angeredet werden, ebenso wie die "hadji" oder Mekkapilger. 